\title{
INVESTIGACIÓN FEMINISTA, HISTORIA DE LAS MUJERES Y MUJERES EN LA HISTORIA, EN LOS ESTUDIOS SOBRE PROXIMO ORIENTE ANTIGUO
}

\section{POR AGNÈS GARCÍA VENTURA}

RESUMO Suele decirse que el estudio del pasado siempre tiene relación con el presente y con el futuro, bien porque presente y futuro se construyen a su imagen y semejanza, bien porque no podemos imaginar un pasado sin los referentes de nuestro presente. Por este motivo, ocuparse de la historia de las mujeres en la Antigüedad y de cómo incluir a las mujeres en la historia, nos permite reflexionar acerca de la situación de las mujeres en el mundo presente en el que vivimos y en el mundo futuro en el que querríamos vivir. En este artículo propongo aproximarnos a este tema con las herramientas críticas de la investigación feminista, ilustrando la propuesta con algunos ejemplos acerca de cómo algunos sesgos pueden afectar al modo en que se aborda el estudio de las vidas de las mujeres en el Próximo Oriente Antiguo.

\author{
FEMINIST RESEARCH, \\ WOMEN'S HISTORY AND \\ WOMEN
}

ABSTRACT As is often said, the study of the past always has to do with the present and with the future, either because present and future are built in its image and likeness, either because we cannot imagine a past without using the references of our present. Therefore, dealing with women's history in ancient times, dealing then with how we can include women in history, allows us to reflect on the situation of women in the world in which we live and in the future world in which we want to live. In this article I intend to approach this issue with the critical tools offeminist research, 
PALAVRAS-CHAVE Investigación feminista

Historia de las mujeres $\underline{\text { Próximo Oriente Antiguo }}$

\section{KEY WORDS}

Feminist research

Women's history

Ancient Near East

Es común la reflexión por parte de quien se dedica a la historia, de que ésta, seal cual sea la época elegida, siempre acaba refiriéndose al presente, al momento en que se escribe. Josep Fontana, historiador de referencia que ha formado a varias generaciones en las universidades de Barcelona, advierte que no hay historia del pasado porque, aunque elijamos una época remota como objeto de estudio, en realidad hacemos historia del presente y para el presente (Fontana 2010: 202). En esta misma dirección, Fontana hace referencia a un pensamiento que el poeta Antonio Machado formuló durante la guerra civil española (1936-1939): "Machado escribió que cuando se examinaba el pasado para ver qué llevaba dentro era fácil encontrar en él un cúmulo de esperanzas, ni conseguidas ni frustradas, esto es, un futuro" (Fontana 2010: 194). Sobre esta idea, aunque planteada de un modo ligeramente distinto, reflexiona también Carol Duncan, en un ensayo sobre los museos, defendiendo su utilidad y vigencia en el siglo XXI. Duncan afirma que sin conocer el pasado no podemos pensar el futuro, pero que sin una idea de futuro tampoco podemos acceder al pasado (Duncan 2007 [1995]: 216).

Vemos, pues, que preguntarse por cuál es la misión y la responsabilidad de quien escribe historia y de quien trabaja de un modo u otro con el pasado y con la transmisión de su legado a la sociedad, es algo habitual. Quizás esto sea especialmente frecuente y pertinente en los contextos sociales en los que se integran los museos, centros de investigación y universidades del primer mundo, es decir las instituciones desde las que buena parte de nosotras llevamos a cabo nuestra investigación, puesto que en estos contextos prima la preocupación por la "utilidad", entendida demasiado a menudo como estrechamente vinculada a la rentabilidad económica. Por consiguiente, se tiende a percibir como prescindible el estudio del pasado, ya sea por considerarlo una actividad inútil o bien por considerarlo como un lujo en un momento de presunta escasez de los recursos económicos que deberían destinarse a sustentar la investigación y la educación.

A mi me parece, sin embargo, que bajo una pátina de aparente despreocupación y descalificación, lo que se oculta es precisamente una preocupación profunda, ya que quien maneja el pasado, quien maneja la historia y la versión que de ella se transmite es alguien que tiene en sus manos cierto poder, pues proporciona los elementos de que se nutre todo imaginario colectivo, toda configuración de una 
determinada identidad. A este particular dedicó Malcolm X unas breves palabras en uno de sus últimos discursos, pronunciado en Nueva York el 24 de enero de 1965 y publicado póstumamente en 1971: "I don't think any of you will deny the fact that it is impossible to understand the present or prepare for the future unless we have some knowledge of the past [...] Just as a tree without roots is dead, a people without history or cultural roots also becomes a dead people" (Malcolm X 1971: 3 y 16).

Precisamente por este motivo yo defiendo que la responsabilidad de quienes nos dedicamos al estudio del pasado es grande, puesto que actuamos como mediadoras y mediadores entre este pasado y la sociedad que debería recibir los resultados de la investigación que hemos llevado a cabo (algo que, dicho sea de paso, sucede con menos frecuencia de la que sería deseable en el ámbito de estudios sobre Próximo Oriente Antiguo). Sea cual sea la época de las que nos ocupamos, son ni más ni menos que los resultados de nuestros trabajos los que aportan los elementos que nutren el imaginario colectivo al que aludíamos antes. Son estos resultados los que a través de las imágenes del pasado que proyectamos ayudan a articular el presente y el futuro. Así pues, estudiar el pasado no supone, desde este punto de vista, buscar unos hechos o una verdad, sino que supone proveer de intepretaciones y lecturas basadas en el escrutinio cuidadoso de las fuentes para así poder (re)construir ese pasado. Como advirtió J. Huizinga ya en 1963, "history gives no more than a particular representation of a particular past, an intelligible picture of a portion of the past. It is never the reconstruction or reproduction of a given past. No past is ever given" (Huizinga 1963: 5).

En referencia al caso específico de los estudios sobre Próximo Oriente Antiguo, como observa Marc Van de Mieroop en uno de los pocos ensayos que existen sobre la relación entre asiriología e historia, parece que en cuanto más lejano es el pasado descrito, más responsabilidad tiene quien escribe historia como mediador o mediadora entre este pasado y la sociedad (Van de Mieroop 1999: 3). A mi entender, asumir esta responsabilidad significa asumir también que, precisamente porque escribiendo historia hacemos un trabajo de mediación, debemos estar muy amatentes a los posibles sesgos, en primer lugar los del propio investigador o investigadora. Acerca del riesgo y de la inevitabilidad de proyectar estos sesgos en nuestra investigación reflexionaba J.J. Finkelstein (1963: 461) en su análisis de la historiografía de los estudios sobre la antigua Mesopotamia:
"In our approach towards any aspect of non-Western civilization we commonly expose ourselves to the hazard of applying Western categories to phenomena completely alien to us. In large measure this is unavoidable, and even necessary; we must convert these phenomena, or translate them, as it were, into our own conceptual language if we are to gain any understanding of them. But we must always be aware of the fact that we are doing so."

Vemos pues, que Finkelstein afirma que es inevitable, en gran medida, llevar a cabo una investigación sin ningún tipo de sesgo. Yo creo, además, que no sólo es 
inevitable, sino poco deseable, ya que, a mi entender, la investigación es siempre política, en el mismo sentido en el que Kate Millet (1969) reivindicó que lo personal es político. Desde esta perspectiva, ciertos sesgos, entendidos como una especie de "discriminación positiva" en la investigación, pueden ser incluso deseables cuando tienen como finalidad defender la justicia social. Por consiguiente se trata, más que de evitar cualquier sesgo, de identificarlos y analizarlos para, así, tratar de contrarestar los que tienen efectos negativos, como la potenciación de la desigualdad entre hombres y mujeres, por ejemplo, en nuestros estudios, favoreciendo en cambio los que pueden tener efectos positivos, actuando pues en dirección contraria.

\section{INVESTIGACIÓN FEMINISTA, HISTORIA DE LAS MUJERES Y MUJERES EN LA HISTORIA}

Vemos pues como al estudiar lo que nos ha precedido en el tiempo ofrecemos siempre nuestra versión de los hechos ante la imposibilidad de relatar "el pasado", un único pasado, puesto que, como hemos visto que advertía Huizinga, "no past is ever given". Podríamos decir, pues, que la plena conciencia de esta influencia del contexto y el derrocamiento de la ilusión de la objetividad son los pilares que sustentan una parte de los modos de escribir historia en la actualidad, en particular aquellos que hacen uso de las teorías críticas, cuyo objetivo no es eliminar todos los posibles sesgos, sino ser conscientes los mismos, tratando a su vez de individuar los que son discriminatorios por algún motivo para favorecer los que promueven, por el contrario, la no discriminación. Se trata, así, de reconocer las diferencias sin darles a las mismas valores jerárquicos distintos que conduzcan a la desigualdad.

A continuación me centraré en una de las opciones que tenemos dentro de las teorías críticas aplicadas al estudio del pasado para conseguir este objetivo: la investigación feminista. En este punto, querría hacer una aclaración acerca de por qué, en este artículo, me referiré a investigación feminista y no a estudios de género. A mi entender, la investigación feminista puede formar parte de los estudios de género, pero no todos los estudios de género podrían calificarse de investigación feminista. Así, mientras que la investigación feminista tiene un claro objetivo político, puesto que pretende denunciar ciertas desigualdades para conseguir rebatirlas y tiene como objetivo la justicia social, los estudios de género incluyen tendencias muy variadas, entre las que hay incluso opciones que difícilmente podríamos calificar de feministas en este sentido político y de intención de cambio de un status quo. Susan Pollock (1999: 218-219), hablando del potencial de la investigación feminista para el análisis del Próximo Oriente Antiguo lo resume del siguiente modo:

\footnotetext{
"A feminist approach considers gender and other socioculturally constructed categories of difference - including class, race, and ethnicity to be central elements in social life. It poses questions about how gender relations, roles, and ideologies, in concert with other forms of difference,
} 
shape and are shaped by social, political and economic change. [...] Both feminism and political economy lead to an appreciation of the complexity of history and historical change."

El principal sesgo que pretende identificar, analizar y derrocar la investigación feminista cuando dota, pues, la historia de más complexidad con la aplicación de estos instrumentos y categorías de análisis, es el androcentrismo. El Diccionario de la Real Academia Española de la Lengua define el término como "visión del mundo y de las relaciones sociales centrada en el punto de vista masculino". Desde la perspectiva de la investigación feminista, habría que añadir a esta definición que el androcentrismo no es "sólo" una visión del mundo, sino que es una visión del mundo que privilegia y coloca jerárquicamente en la cima el punto de vista construido como masculino en detrimento de ciertos puntos de vistas, actividades, etc. asociadas a lo femenino. Contrarestar el androcentrismo en el ámbito que aquí nos ocupa, pues, supone contrarestar una desigualdad: la desigualdad entre lo que se asocia a los hombres y las masculinidades (considerado jerárquicamente superior) y lo que se asocia a las mujeres y a las feminidades (considerado jerárquicamente inferior) en algunos estudios de historia del Próximo Oriente Antiguo.

Cuando me refiero aquí a hombres, masculinidades y punto de vista masculino versus mujeres, feminidades y punto de vista femenino no aludo a cualidades ni características innatas en los hombres o en las mujeres. Mi punto de partida es constructivista y no esencialista, de modo que no creo que podamos adjudicar características innatas diferenciales a hombres y a mujeres. Sí me parece claro, sin embargo, que necesitamos estos conceptos para usarlos como categorías analíticas (y quizás para ser convenientemente deconstruidos en una fase posterior de la investigación) puesto que, durante décadas, han sido los ejes alrededor de los que la investigación ha girado. Si hasta ahora se han abordado temas de estudio en historia antigua como el trabajo de los hombres o de las mujeres, la división sexual de este trabajo, las categorías laborales, la familia o las relaciones sexuales, es necesario analizar cómo en cada uno de estos temas se ha hablado de hombres, mujeres, feminidad(es) y masculinidad(es) para así poder identificar posibles sesgos androcéntricos.

Así pues, la idea es dar una vuelta de tuerca más al que fue el primer objetivo de la investigación feminista ya en los años 70 del siglo XX: "buscar mujeres" en la historia, algo que también se denominó en algunos estudios posteriores de la "gender archaeology" como el "añadir mujeres y agitar" (Tringham 1999 [1991]: 100). El caso es que, tras "buscar mujeres", éstas fueron "añadidas" en una "historia de las mujeres", como si ellas no formaran parte de la foto fija, del panorama general que se dibuja a partir de las fuentes cuando se intenta reconstruir lo que llamamos "historia". Pasadas ya unas cuantas décadas desde el nacimiento de los estudios feministas en sus diferentes tendencias, quizás sea ya el momento de conseguir que, cuando algunas de nosotras decimos que nos dedicamos a estudiar las vidas de las mujeres no se nos diga "ah, sí, muy interesante... pero esto sólo os interesa a las mujeres..." (Scott 1999: 30-31). El objetivo debe ser que nuestros 
estudios alcancen el interés general del que durante siglos han gozado algunos temas que, siendo propios en muchas ocasiones de esferas predominante o exclusivamente masculinas, se han presentado como temas de interés general (Juliano 1998: 7). Y para ello se trata de construir discursos generales en los que las mujeres también quepan, dando pues un paso más allá del "añadir mujeres y agitar".

En otras palabas, poner de relieve las vidas de las mujeres es, a mi entender, un punto de partida, no de llegada. Al igual que sucede con las reivindicaciones de algunas minorías étnicas, se trata de analizar la diferencia con el fin de, asumiéndola y normalizándola, acabar con las barreras y los obstáculos y con las valoraciones jerárquicas de esta diferencia. Como dijo hace ya algunos años Donna Haraway, "Una feminista es alguien que lucha por las mujeres en tanto que clase y por la desaparición de esa clase" (Haraway 1995 [1991]: 233).

Así pues, aunque el "buscar mujeres" sigue siendo necesario en los estudios sobre Próximo Oriente Antiguo, creo que es también deseable combinar o completar este objetivo, que conduce a escribir una historia de las mujeres, con la idea de integrar a las mujeres en la historia. Esto es especialmente necesario en asiriología, donde son pocos los trabajos que consideran el género como categoría de análisis. No obstante, es pertinente observar que cuando pretendemos aplicar ciertos marcos teóricos como los propuestos desde algunas teorías críticas a los estudios asiriológicos debemos actuar con cautela. La tipología de las fuentes, sus limitaciones y la conciencia de que las problemáticas de la identidad y el sujeto de la modernidad y la postmodernidad no son las mismas que en la antigua Mesopotamia hacen que debamos tener esta precaución (Bahrani 2001: 25-27).

En cualquier caso, mi propuesta aquí es partir es partir de un feminismo de la diferencia, por así llamarlo, para llegar a un feminismo de la igualdad que haya incluido las diferencias (no que las haya minimizado o eliminado) para conseguir tal igualdad. Se trata sin duda de un fenómeno complejo que no podemos alcanzar sólo con nuestras investigaciones sobre el pasado, pero al que sí creo que podemos intentar contribuir. A este respecto es muy ilustrativo el Manifiesto para cyborgs de Donna Haraway (1995 [1991]: 254), que en la definición de sus objetivos plantea lo siguiente:

"El presente trabajo es un canto al placer en la confusión de las fronteras y a la responsabilidad en su construcción. Es también un esfuerzo para contribuir a la cultura y a la teoría feminista socialista de una manera postmoderna, no naturalista, y dentro de la tradición utópica de imaginar un mundo sin géneros, sin génesis y, quizás, sin fin." 
Para construir tanto la historia de las mujeres como una historia en la que las mujeres quepan considero necesario identificar, analizar y tratar de contrarestar los sesgos androcéntricos, como apuntaba en la sección anterior. Para tal fin son especialmente útiles las propuestas lanzadas desde las llamadas "epistemologías feministas” (Alcoff \& Potter 1993), surgidas en ámbito anglosajón en los años 80 del siglo XX, con una reflexión acerca de los conocimientos situados, un tema que no era nuevo. Ya Hannah Arendt, en los años cincuenta, planteó el tema de los conocimientos situados en su ensayo sobre el concepto de historia, cuando explicitó que tanto esta disciplina como la física son subjetivas (Arendt 2003 [1954]: 80-81). No en vano, en los años ochenta, serán algunas científicas las que pondrán el tema sobre la mesa.

En cuanto a los objetivos de las epistemologías feministas, el primero es llamar la atención sobre el androcentrismo presente en la investigación. En este sentido, esta propuesta teórica, que podríamos situar en el marco del llamado postfeminismo, es comparable al postcolonialismo: si el postcolonialismo alerta acerca del etnocentrismo, el postfeminismo hace las veces respecto al androcentrismo. Y como se ha demostrado desde los estudios postcoloniales ampliamente, para alertar acerca del etnocentrismo (o del androcentrismo para el caso del postfeminismo) y combatirlo de manera efectiva es necesario proponer nuevos marcos teóricos que permitan cuestionar el modo en que se han planteado las investigaciones precedentes, ya que como dijo Audre Lorde en el título de su conferencia más famosa, pronunciada en Nueva York en 1984, "las herramientas del amo nunca desmantelarán la casa del amo".

Así pues, este es, precisamente, el segundo de los objetivos principales de las epistemologías feministas, que pretende cuestionar y repensar cómo se ha dado el proceso de producción del conocimiento. En particular, se pretende analizar cómo y por qué se han elegido durante siglos los temas de estudio y cómo se han planteado las preguntas de investigación, para así poder trazar estrategias que permitan incluir a las mujeres en los discursos históricos y que permitan, a su vez, evitar las ideas preconcebidas que han conducido a interpretaciones y conclusiones de claro sesgo androcéntrico. Para alcanzar estos objetivos, desde las epistemologías feministas se han propuesto varios marcos teóricos. A continuación presento brevemente los que tres de sus teóricas más representativas propusieron durante la década de los años 80 del siglo XX: Nancy Hartsock, Sandra Harding y Donna Haraway.

Haciéndose eco de la mañida dicotomía "hombre-cultura versus mujer-naturaleza", la filósofa feminista Nancy Hartsock formuló un provocativo contraste entre lo que ella convino en llamar "masculinidades abstractas" y el "punto de vista feminista" (Hartsock 1983). Hartsock usa el psicoanálisis para explicar cómo en los hombres el yo se construye entorno a lo abstracto, mientras que en las mujeres se construye a través de objetos y de experiencias tangibles. Como resultado de este proceso diverso de construcción de la identidad, las mujeres tenderían a estar más apegadas a y más condicionadas por las experiencias de la cotidianidad y por sus cuerpos. Además, la masculinidad tiende a presentarse como "neutra" o invisible, mientras que la feminidad tiende a presentarse como construida, marcada y, en consecuencia, 
visible. Al respecto, Hartsock denuncia que mientras que la "masculinidad abstracta" se considera fundamental para trazar el panorama general de la historia, el "punto de vista feminista", precisamente porque se presenta como un punto de vista específico y concreto, se considera como algo que puede afectar sólo en algunos casos particulares, y que por lo tanto no merece ser considerado en la mayor parte de las ocasiones cuando se trata de dibujar el panorama general al que aludíamos antes.

Otra de las teóricas de las epistemologías feministas, la filósofa y bióloga Sandra Harding propuso lo que ella llamó "epistemología del punto de vista" (véase su publicación de 1986 para su primera formulación de la propuesta [traducción al castellano de 1996] y cf. con Harding 2012 para sus ulteriores reflexiones acerca de la recepción de su propuesta). El núcleo de la propuesta defiende que quienes están en posiciones subordinadas tienen un punto de vista privilegiado, ya que su punto de vista es, por una serie de motivos que desgrana Harding, menos sesgado que el de sus opresores en relación a ciertos asuntos. Como Harding constataba que las mujeres estaban en posiciones subordinadas en muchas geografías y cronologías, para ella sus puntos de vista debían ser considerados privilegiados.

En parte como reacción a la propuesta de Harding, la historiadora de la ciencia Donna Haraway (una de las voces principales del ciberfeminismo), lanzó la propuesta conocida como "conocimientos situados" en un artículo en la revista Feminist Studies (1988, propuesta ratificada en su Ciencia, cyborgs y mujeres [traducción al castellano de 1995 de su volumen original en inglés de 1991]). Haraway reivindicó que no es posible llevar a cabo una investigación de manera neutra $u$ objetiva, puesto que nuestra investigación está siempre sesgada por nuestro punto de vista, un punto de vista que no podemos eliminar por completo, ni tan sólo en las disciplinas científicas como la biología, a la que ella se dedica. Así pues, para Haraway la "objetividad feminista" no puede ser otra que la del conocimiento situado (Haraway 1995 [1991]: 324; cf. Fox Keller 1991), que alcanzamos desde nuestra propia y particular parcela, es decir, con influencias del lugar en el que hemos nacido, del lugar en el que vivimos, de nuestro género, de nuestra edad, de nuestro estatus, etc.

Vemos pues que, pese a algunas discrepancias (que yo considero menores), las propuestas de Hartsock, Harding y Haraway aquí resumidas tienen muchos puntos en común. Básicamente, todas pretenden dar herramientas para que podamos detectar cómo nuestras ideas preconcebidas afectan al modo en que llevamos a cabo nuestra investigación, a la elección de los temas de estudio, etc., herramientas, pues, para desmantelar la casa del amo, si nos hacemos eco de las palabras de Lorde. Por este motivo, estas propuestas se conocen también como defensoras de lo que se ha convenido en llamar "objetividad fuerte" ("strong objectivity"), que pretende poner en evidencia la falacia del uso habitual de "objetividad" entendida como un modo de producir conocimiento que es capaz de ser neutral y de mostrarse como absolutamente desvinculado del cuerpo. 
Al respecto es muy pertinente el modo en que el arqueólogo Bernard Knapp (1996: 145) reflexionó acerca de estos asuntos y acerca de su influencia en el modo que tenemos de llevar a cabo nuestra investigación, refiriéndose en particular al estudio del pasado:

"The real problem lies not with those who write from a particular viewpoint - be it political, Green, ethnic, sexist, masculinist, or feminist since they make clear their points of view; the problem lies with those who believe they are writing objective, apolitical, ideology-free archaeology."

\section{DESIGUALDAD Y SESGOS ANDROCÉNTRICOS EN ASIRIOLOGÍA}

Veamos ahora algunos ejemplos de cómo aplicar los planteamientos de las epistemologías feministas, partiendo de su idea de la "objetividad fuerte", nos ayuda a detectar el sesgo androcéntrico presente en en algunos estudios asiriológicos. Como señalaba anteriormente, detectar este sesgo es algo fundamental para contrarestar la desigualdad entre hombres y mujeres que, a menudo, se reproduce y se fomenta, de manera inconsciente (cf. Hernando 2012: 16-17), tanto en la elección de los temas de estudio como en el modo de aproximarnos a los mismos en nuestra disciplina.

A continuación mostraré ejemplos de casos de estudio en los que los puntos de partida son, a mi entender, androcéntricos. Y para ello me centaré en dos de estos posibles puntos de partida. En primer lugar, me ocuparé de los que presentan a las mujeres como indefectiblemente vinculadas y condicionadas por sus cuerpos, mientras que asocian a los hombres a lo abstracto. En segundo lugar, me detendré en los que asumen la existencia de una clara separación entre la esfera de los hombres y la esfera de las mujeres y que, por ello, tratan de encontrar, en los casos de estudio, trazas de esta neta separación, vista como eje central que articula y divide la sociedad.

\section{1. "MASCULINIDADES ABSTRACTAS" VERSUS "PUNTO DE VISTA FEMINISTA" EN ASIRIOLOGÍA}

Empecemos por un par de ejemplos de cómo mostrar a las mujeres como completamente condicionadas por sus cuerpos puede llevar a resultados sesgados. Para ello pondré sobre la mesa, primero, una monografía reciente de Marten Stol (2012) y a continuación me centraré brevemente en cómo entre 1988 y 2008 se ha estudiado una de las cartas de Mari (actual Tell Hariri).

Marten Stol publicó en 2012 su esperada monografía sobre las mujeres en el Próximo Oriente Antiguo (ya anunciada, por ejemplo, en un artículo suyo de 1995). 
La monografía, hasta ahora sólo publicada en holandés, se ocupa de los siguientes temas (listo a continuación mis traducciones aproximadas del holandés al castellano de los temas tal y como aparecen en el índice): el matrimonio, la familia y la herencia, mujeres privadas de libertad, mujeres trabajadoras, brujas, prostitutas y prostitutas sagradas, integridad física y vida (incluyendo enfermedad, vejez y muerte), la corte y el harén, sacerdotisas, conventos, lo sagrado, adivinación y, finalmente, matrimonio sagrado. Viendo este índice (y el tratamiento que hace Stol de los temas en él apuntados), hay varios detalles que merecen un comentario. El primero, que Stol dedique sólo 20 páginas (de las 480 de la monografía) a las mujeres trabajadoras, páginas en las que él incluye a músicas, molineras, tejedoras, plañideras y emprendedoras, mientras que dedica dos capítulos a la corte y el harén; o incluso cinco capítulos a la relación entre las mujeres y lo "ritual" o "sacro" (sacerdotisas, conventos, lo sagrado, adivinación, matrimonio sagrado). También es interesante, viendo cuán breve es el capítulo dedicado a las mujeres trabajadoras, que dedique capítulos a parte a las brujas y a las prostitutas que, a la vista está, no se consideran trabajadoras según su propuesta. Por otra parte, constatamos que prácticamente todos los temas muestran a las mujeres como condicionadas por sus cuerpos, puestos éstos están en el centro de todo el discurso y por lo tanto en el centro de cómo se presentan estas mujeres.

Pasemos ahora al segundo ejemplo, el estudio de una carta de la antigua ciudad de Mari (Tell Hariri), ciudad situada cerca de la frontera entre Irak y Siria. De este yacimiento provienen numerosas tablillas datadas entre el final del siglo XIX y mediados del siglo XVIII a.n.e., siendo muchas de ellas cartas. Una de estas cartas (A.2679 = ARM 26, 13), escrita por Ašqudum, oficial al servicio de Yarim-Lim, el rey de Aleppo, se refiere a la llegada de la hija de Yarim-Lim, Šibtu, a la ciudad de Mari, donde ésta se casará con Zimri-Lim, el rey de dicha ciudad. La carta, que publicó por primera vez en transliteración y en traducción Jean-Marie Durand en 1988, ha sido comentada en varias ocasiones, en varios estudios, entre 1988 y 2008, poniendo a menudo de relieve cómo, según algunos estudiosos, en la misma se habla de la menstruación y de sus implicaciones en términos de pureza / impureza. A analizar este particular se dedica el artículo conjunto de Érica Couto-Ferreira y Agnès Garcia-Ventura, publicado en la revista Gender \& History en 2013, donde se puede encontrar una presentación detallada de los argumentos y referencias que aquí sólo comentaré brevemente.

Empecemos mostrando un fragmento de la propuesta de traducción de Durand, que sería la traducción de referencia, publicada en 1988 (y también citada en una publicación suya anterior de 1987: 102): "Il a dit: «Que les affaires de ma fille soient (donc) déposées dans sa demeure, (mais) que ma fille réside chez son époux et que 5 ou 6 jours elle quitte (le palais) et qu'elle s'ocupe de sa demeure»" (Durand 1988: 110-111). Vemos que en este fragmento se habla de que la princesa se vaya durante 5 o 6 días de algún lugar y se ocupe de sus cosas, sin ninguna alusión a la menstruación ni a nada similar, aunque en el comentario de la carta, Durand (1988 : 105-106) añade un "par mois", entre paréntesis, después de mencionar los cinco o seis días en que la princesa deberá abandonar el palacio. 
También en 1987 Bertrand Lafont cita esta carta en un estudio y hace un pequeño añadido a la traducción (pequeño pero muy significativo) del "par mois" que en el caso de Durand sólo aparecía en el comentario, pero no en la traducción. Este añadido, junto con la mención del "par mois" en el comentario de Durand, ayudan a que, de aquí en adelante, se de un giro de 180 grados en la interpretación de la carta: "5 à 6 jours par mois qu'elle se retire chez elle" (Lafont 1987: 24; la negrita es mía). Este "par mois", se interpreta a partir de ese momento, por buena parte de los estudiosos y las estudiosas, como una alusión a la menstruación de la princesa, lo que lleva a que en la literatura secundaria posterior encontremos generalizaciones como las siguientes, que podemos leer en publicaciones de Nele Ziegler (1999) y de Adelina Millet (2008) respectivamente: "il semble que les femmes avaient l'habitude de quitter le palais pour la durée de leur règles" (Ziegler 1999: 32) // "On sait per ailleurs, grâce notamment au corpus de Mari, que les femmes résidant dans le harem et, plus largement, dans le palais, le quittaient au moment de leurs menstrues, pur des raisons d'impureté rituelle" (Millet 2008: 247). Huelga decir que, estas generalizaciones, basadas sólo en un texto y en un añadido de dos palabras (“cada mes"), son claros ejemplos de cuán sesgada puede ser la interpretación de un texto cuando el punto de partida es ya sesgado.

A mi entender, tanto el caso de la monografía de Stol como el de la carta de Mari que presuntamente se refiere a la menstruación, son buenos ejemplos de estudios que, como hemos visto en la sección previa, tienen como punto de partida lo que Hartsock formuló de manera provocativa contrtaponiendo las "masculinidades abstractas" al "punto de vista feminista". En algunos estudios que se dedican a la historia de las mujeres, éstas se presentan como "otro", como objetos de estudio y no como sujetos, algo que se materializa en algunos casos presentando a las mujeres como completamente condicionadas por sus cuerpos. Estos puntos de partida son los que explican que, a menudo, escribir historia de las mujeres se identifique con hablar exclusivamente (o casi) de parentesco (definido sólo a partir de vínculos biológicos y sexuales, como sería el caso de la filiación y el matrimonio) y de todo aquello que tiene que ver de manera directa con las especificidades de los cuerpos de las mujeres, manifestadas en la gestación, el parto, la menstruación, dicho de otro modo, todo lo que se relaciona con la maternidad y la fertilidad, temas que, a su vez, se tiende a interpretar en clave ritual, poniendo en juego conceptos como los de pureza / impureza ritual o sexo vinculado a la esfera ritual (como sería el caso de lo que se conoce como "prostitución sagrada" o el "matrimonio sagrado", temas que se han estudiado profusamente en asiriología con claros sesgos androcéntricos, como demuestra de manera brillante, por ejemplo, Stephanie Budin en su monografía de 2008 en la que se ocupa de la presunta prostitución sagrada).

En el caso de los hombres, en cambio, suelen tratarse temas más relacionados con los vínculos de afinidad que con los de consanguineidad, de modo que en lugar de poner en el centro las relaciones sexuales o el parentesco, se da protagonismo a sus acciones políticas, sus relaciones diplomáticas, su vida laboral e intelectual, temas que rara vez ponen en el centro el hecho de que los hombres puedan o no estar condicionados por sus cuerpos (cf. Hernando 2012), algo que, a mi entender, es la 
clara muestra de que, cuando se eligen ciertos temas al escribir historia de las mujeres, ello supone ya un punto de partida que contiene un claro sesgo androcéntrico, que, a su vez, puede ser reforzado con el enfoque que se de a estos temas. En otras palabras, que se trata de un sesgo que fomenta la desigualdad entre hombres y mujeres es para mi fácilmente demostrable si, cuando pensamos en un tema parece "apto" para escribir historia de las mujeres pero no para escribir "Historia" en mayúsculas. ¿Imaginamos un manual de historia del Próximo Oriente Antiguo con un índice como el de la monografía de Stol? Seguramente no, y defiendo que escudriñar por qué no puede ser una buena manera de evidenciar cietos sesgos para así proceder a eliminarlos.

\section{2. ¿JUNTOS PERO NO REVUELTOS? MUNDOS DE HOMBRES VERSUS MUNDOS DE MUJERES}

En esta sección mostraré algunos ejemplos en los que se asume la existencia de una clara separación entre la esfera de los hombres y la esfera de las mujeres, algo que, si se toma como punto de partida, veremos que puede también reforzar el sesgo androcéntrico, porque defiendo que cuando se habla de esferas tan netamente separadas se tiende a dar más valor a lo asociado a los hombres y menos valor a lo asociado a las mujeres. Sin embargo, paradójicamente, esta premisa ha sido utilizada, en muchos casos, precisamente con finalidades feministas, en especial en el marco de lo que se conoce como la primera ola del feminismo académico, que tenía como objetivo visibilizar a las mujeres, por lo que creo que su detección es especialmente interesante y pertinente. Primero comentaré brevemente cómo se trata este particular en un artículo reciente de Harriet Crawford (2014) y a continuación presentaré algunas reflexiones sobre el uso de la división sexual del trabajo como punto de partida habitual para el estudio del trabajo, en especial cuando se pretende analizar el trabajo de las mujeres.

En el año 2014 Mark Chavalas editó el volumen titulado Women in the Ancient Near East. A Sourcebook, en el que varias estudiosas presentan en traducción y comentario fragmentos de textos de tablillas cuneiformes, de entre finales del tercer milenio y finales del primer milenio a.n.e., que nos ayudan a aproximarnos a las vidas de las mujeres a través de los textos producidos durante cerca de 2000 años (para una recensión del volumen, véase Svärd 2015). En el primero de los artículos, de Harriet Crawford, se presentan las fuentes para el tercer milenio a.n.e. y, aunque queda claro desde el inicio que la autora advierte cuán perjudiciales pueden ser los sesgos androcéntricos para la investigación (v. Crawford 2014: 10-11 para su crítica del "orientalismo" en temas de estudio como el velo femenino o el presunto harén en la Antigüedad, basados ambos en analogías etnográficas), ella misma toma como punto de partida lo que yo interpreto precisamente como uno de estos sesgos: la existencia de la clara separación entre el mundo de los hombres y el mundo de las mujeres en el tercer milenio a.n.e. 
Crawford, muy posiblemente en la línea de lo que se conoce como feminismo de la diferencia al que me he referido antes, no considera que este punto de partida sea androcéntrico, al contrario: puede ser leído como una muestra del empoderamiento de las mujeres en ciertos ámbitos, como veremos a continuación. En particular, la estudiosa trata el tema del Emesal, la presunta "lengua de las mujeres" sumeria, como evidencia de cuán separados están estos mundos, que incluso necesitan una variante lingüística para cada uno de ellos. En primer lugar, llama la atención en un volumen articulado a partir de la cronología que trate este tema en un artículo dedicado al tercer milenio, puesto que como ella misma bien observa, el Emesal no se atestigua en el tercer milenio, sino a inicios del segundo milenio a.n.e. (Crawford 2014: 20). En segundo lugar, aunque sigue siendo habitual en la literatura secundaria referirse al Emesal como la "lengua de las mujeres", el debate está abierto y ahora mismo son más los y las especialistas que no lo ven como tal, de manera que tomarlo como una evidencia no es adecuado (Michalowski 2004: 23).

El párrafo que nos deja entrever de manera más elocuente cuál es la premisa de su investigación es el siguiente, en el que Crawford (2014: 21) defiende que los contactos entre hombres y mujeres eran tan escasos que se daban básicamente con fines procreativos y poco más:

"It should be stressed again that neither the male nor the female world was exclusive to one gender or another, men and women worked in both spheres, and men could be subordinate to women in the women's world. It can further be suggested that there was relatively little contact between the two worlds, except for the purposes of procreation and on certain formal occasions, although no doubt love matches occurred; this is another area that needs exploring. Evidence from recent anthropological work on women in traditional Arab societies seems to show a similar pattern."

Otro ejemplo clásico en el que vemos que el punto de partida es la existencia y separación de lo que se consideran mundos de hombres y mundos de mujeres es la categoría de análisis que se conoce como división sexual (o de género) del trabajo (en adelante me referiré sólo a "división sexual" y no a "división de género"; para el argumentario a favor y en contra del uso de ambas denominaciones, véase Garcia-Ventura, en prensa). La división sexual del trabajo parte de la premisa de considerar que el hecho de que algunas actividades sean realizadas exclusiva o mayoritariamente por hombres, por mujeres o de modo equitativo por ambos es significativo. Así, cuando aplicamos esta división, partimos del supuesto de que, al estudiar las fuentes primarias, encontraremos hombres o mujeres llevando a cabo ciertas tareas, dando por sentado, a su vez, cuáles son las tareas más habituales para los unos o para las otras.

Por poner un par de ejemplos clásicos, suele asociarse de manera general y casi automática en muchos estudios el tejido a las mujeres y la metalurgia a los hombres (dándose así a priori una división sexual del trabajo basada en la especialidad); asimismo, suelen también asociarse los puestos de mando a los hombres y los subordinados a las mujeres (dándose así, de nuevo a priori, una división sexual del 
trabajo basada en la jerarquía). Cuando se asumen estas premisas como puntos de partida para la investigación, o bien se buscan en las fuentes evidencias de estas asociaciones o bien, cuando no se encuentran de manera explícita, suele darse por sentado que la asociación se dio de esta manera, algo que raramente es cuestionado. Para mi es necesario abandonar estos prejuicios para poder ver que esta división, en algunos casos, no responde a lo que se atestigua en las fuentes si las analizamos atentamente y sin tomar estos puntos de partida. Además, me parece también importante analizar por qué estos prejuicios se dan y por qué son tan persistentes pese a que a menudo el estudio de las fuentes primarias no ayuda a confirmarlos.

Una posible explicación es la aceptación de lo que se ha denominado "contrato sexual", que surge de una lectura determinada de cómo se establece el vínculo entre producción, reproducción y división sexual del trabajo. Esta lectura determinada propone que la distribución de tareas entre mujeres y hombres sería un modo de asegurar, para las mujeres, protección a cambio de subordinación a los hombres. Se trataría pues de un "contrato sexual" comparable al "contrato social" (Nuño Gómez 2010: 37-56, en especial p. 45 para este argumento). De este modo, los trabajos considerados mayoritaria o exclusivamente femeninos han tendido a leerse como subordinados o dependientes de otros mayoritaria o exclusivamente masculinos, algo que a mi entender es un claro ejemplo de cómo opera el sesgo androcéntrico.

En este sentido, Sarah Milledge Nelson (1997: 85-88) alertó acerca de cuáles son las ideas preconcebidas que subyacen el uso de la división sexual del trabajo como categoría de análisis, de las que destaca dos. En primer lugar, la estudiosa notaba que en este marco teórico, la categoría de análisis "mujer" se usaba a menudo como equivalnte de "madre", dando por sentado que los roles de la maternidad condicionan todas las elecciones laborales y profesionales de las mujeres durante toda su vida. En segundo lugar, Nelson nota que "the division of labor is absolute: if men do it, women can't, or don't, or are prohibited from doing it, and vice versa" (Nelson 1997: 86). Si lo que queremos es dotar de complejidad nuestros análisis y relatos del pasado, claro está que estas ideas preconcebidas acaban consiguiendo precisamente lo contrario, puesto que sustentan explicaciones unívocas y unidireccionales, es decir simples y no suficientemente complejas (cf. Chapman 2010 [2003] para el caso de las llamadas "arqueologías de la complejidad") al retratar divisiones tan claras entre las actividades que llevan a cabo los hombres y las que llevan a cabo las mujeres.

Por consiguiente, defiendo que partir de estas ideas preconcebidas acerca de cuáles son las ocupaciones de los hombres y cuáles las de las mujeres, dando pues por sentado que el sexo y el género son los ejes fundamentales a partir de los que se articula la organización del trabajo (olvidando así edad, especialidad, rango), puede llevarnos a resultados de investigación sesgados. Un buen ejemplo es el caso de los cargos de supervisión en el sector textil tanto en el Lagaš Presargónico como en la Tercera Dinastía de Ur (ambos períodos situados en el último cuarto del tercer milenio a.n.e.), donde se suele dar por sentado que los hombres son los capataces, de modo que cuando tenemos evidencias de alguna mujer que ostenta tal cargo, 
viene presentada como una anomalía, como algo excepcional que es necesario justificar.

Sin embargo, no debió ser tan excepcional, puesto que tenemos evidencias en los textos y la glíptica de la existencia de algunas mujeres que llevaron a cabo estas tareas de supervisión. En los textos de Garšana, fechados en la Tercera Dinastía de Ur, se mencionan supervisoras como Aštaqqar, un nombre propio femenino que aparece asociado a ugula uš-bar (capataz o capataza de la tejeduría en sumerio). Del ejemplo de Aštaqqar sería de especial interés, a mi entender, que un nombre propio que se identifica como femenino vaya asociado a dos términos ambiguos en cuanto al género gramatical (neutro en los nombres sumerios si no se especifica que se trate de hombre / mujer o masculino / femenino) (para el desarrollo detallado de este argumento y de otros casos de estudio, véase Garcia-Ventura 2013). Por otra parte, en lo referente al Lagaš Presargónico, se pueden reconocer, por los pagos que reciben y las tareas que se les asignan, mujeres que ostentan el cargo de capatazas aunque no se identifiquen con el término sumerio ugula (recordemos, neutro en cuanto al género gramatical se refiere), algo que ha llevado a diversos estudios a no considerar a estas mujeres como capatazas, negándoles así una jerarquía que se presupone, de entrada, masculina (sobre este asunto, con ejemplos específicos de textos y de capatazas, véase Karahashi \& Garcia-Ventura, en preparación).

Viendo estos dos ejemplos, pues, defiendo que sólo si no partimos de una idea preconcebida acerca de quién lleva a cabo qué tarea o bien quién ostenta ciertos cargos, podremos dilucidar, sin la influencia distorsionadora de los sesgos androcéntricos, quién la llevaba a cabo efectivamente y también si el sexo o el género eran factores centrales para asignar ciertas tareas a ciertas personas o si, en cambio, simplemente eran un factor más a tener en cuenta (aunque no el central) o incluso un factor que no era relevante en ciertos contextos.

\section{ACERCA DE LA RELACIÓN ENTRE HISTORIA Y ASIRIOLOGÍA: REFLEXIONES Y RETOS}

En este artículo he tratado de mostrar cómo tratar de identificar el sesgo androcéntrico presente en algunos estudios, tal y como proponen las epistemologías feministas, puede ayudarnos a reducir la desigualdad entre hombres y mujeres que a veces se refuerza on incluso se construye en la literatura secundaria. Con ello, pues, defiendo que estaremos en condiciones de establecer unos vínculos más fructíferos entre pasado, presente y futuro, algo que no es baladí por la repercusión social que conlleva, tal y como apuntaba en la primera sección.

Al respecto, el camino por recorrer es todavía largo, en especial en asiriología, una disciplina todavía muy encerrada en sí misma. Muestra de este aislamiento de la disciplina es el hecho de que sea difícil encontrar obras de divulgación de algunos temas (todavía más en lenguas como el castellano), textos (en especial sumerios) publicados en traducción a alguna lengua moderna, o incluso obras generales que 
sean inteligibles para quienes no son especialistas en uno u otro periodo de la historia mesopotámica, aunque sean profesionales de la historia. Muchos de los trabajos son puramente filológicos, con lo que la historia propiamente dicha es escasa. En asiriología esto sucede, por una parte, porque el positivismo está todavía muy presente en la disciplina; por otra parte, por algunas particularidades y dificultades propias de este campo de estudio.

Pongamos un ejemplo. En asiriología es común que el o la especialista de un periodo concreto no pueda leer los textos de otro periodo, pese a estar fijados con la misma escritura e incluso con la misma lengua. Las variaciones de los signos, de las palabras mismas o de su sentido entre distintos periodos son algunas de las circunstancias que provocan una extrema especialización en asiriología. Este fenómeno, muy patente en nuestra disciplina, es también uno de los problemas generales de una investigación que cada vez tiende más a la especialización hasta perder, en algunos casos, la visión global, algo necesario para describir ciertos procesos históricos (Fontana 1992: 18). También Mario Liverani, uno de los pocos historiadores del Próximo Oriente Antiguo, puesto que la mayoría de especialistas son puramente filólogos o arqueólogos, se lamentaba ya de este asunto en la primera edición de su manual Antico Oriente en 1988 en un primer capítulo titulado "L'antico Oriente come problema storico" (Liverani 1988: 5-20). Parece que en los últimos casi 30 años el panorama ha cambiado poco (cf. Liverani 1999).

A mi entender, si no llega a producirse esta historia, pues, se rompe el vínculo entre quien hace el trabajo de investigación y la sociedad. Así, esa responsabilidad del historiador o de la historiadora a la que aludíamos, desaparece, algo que no me parece para nada positivo. Por otra parte, esta desvinculación está también parcialmente relacionada con el carácter decimonónico que todavía hoy en día tiene la disciplina. Algunos asiriólogos y asiriólogas como Marc Van de Mieroop (1999: 3), Aage Westenholz (2006) o Andrea Seri (2012: 9-28) han hecho algunas observaciones al respecto. Westenholz incluso se pregunta, con el título de su artículo publicado en 2006, si la asiriología tiene un futuro. La pregunta, para algunas de nosotras, puede ser inquietante, pero más lo es la respuesta que Westenholz propone: depende. Depende de si la asiriología es capaz de dejar de funcionar como si todo dependiera todavía del mecenazgo del siglo XIX, es decir, siendo consciente de para quien escribe hoy en día, quienes son sus "clientes", quién es su público potencial. Y ahí entran de nuevo en juego las mujeres, excluidas demasiado a menudo, de un modo u otro, de los discursos históricos que se han construido.

Y es que quizás ahora mismo, a principios del siglo XXI, la historia de la antigua Mesopotámica esté contra la pared. Por un lado, tiene todavía el peso de una disciplina decimonónica que, como hemos descrito, debe todavía aligerarse para que se de un cambio profundo en los temas elegidos para el estudio y su enfoque. Por el otro, y posiblemente esta sea hoy en día la fuerza que la empuja contra la pared anterior, quienes tratamos de movernos en la pantanosa disciplina histórica, más si cabe cuando tratamos de llevar a cabo investigación feminista, corremos el peligro de caer en las garras del relativismo puro. Nos enfrentamos a la 
imposibilidad de escribir historia porque desde el conocimiento situado la historia no "existe", no está ahí para ser tomada, sino que debemos construirla y reconstruirla, una y otra vez.

Al respecto, Michael Heyd, en una reciente reflexión acerca de si tiene futuro el estudio del pasado, alerta de que el problema es que nos movemos sólo en los extremos, y debemos encontrar la gama de grises. Si esto sucede en la historia en general, como disciplina, quizás todavía más en asiriología, donde dadas las dificultades que se plantean al trabajar con las fuentes escritas es más fácil, si cabe, caer en el relativismo cuando tomamos plena conciencia de las limitaciones de estas fuentes. Heyd (2012: 2) plantea la siguiente diagnosis y propuesta:

"Personally, I think that this sceptical critique has gone much too far and I am worried by the extent to which some practicing historians have accepted that radical critique. In between a naïve view of «objective», «scientific» history, and a sceptical view which sees all historical narratives as just another type of fiction, I believe that there is a vast territory of critical discourse which can and should sustain responsible historical scholarship."

Para Heyd es en parte por este relativismo por lo que la historia ha dejado de parecer útil a la sociedad. Si no encontraremos en la historia una verdad revelada, unos hechos precisos y que sin duda se dieron, entonces la historia deja de tener, a ojos de la sociedad actual, esa misión de crear raíces e identidad a la que aludía Malcolm X en el discurso al que hemos aludido al inicio. Además, como también observa Heyd en este mismo artículo, en las últimas décadas quienes escriben historia han estado muy ocupados y ocupadas en estar a la última, en hacer piruetas teóricas, olvidando una vez más que no sólo a los y las especialistas se dirige lo que producimos. Así pues, también desde la investigación feminista y desde los estudios de género es necesaria una labor de autocrítica en este sentido.

En cualquier caso, lo que propongo aquí es llevar a cabo un análisis crítico, que explícitamente use propuestas teóricas determinadas, para así romper la ilusión de la objetividad que todavía está muy presente en asiriología. Tras este primer paso, será entonces posible pasar a plantear hipótesis de lectura de los datos que no deberían ser vistas como más especulativas o subjetivas que las traducciones mismas de los términos, ya que ambas fases del trabajo son subjetivas y no, como suele verse, objetivo el trabajo con los textos, subjetiva sólo la interpretación de los mismos. En el caso de las tablillas cuneiformes, y muy especialmente las que están escritas en lengua sumeria, esta labor de crítica es compleja, ya que las herramientas necesarias para realizarla son, de por sí, de difícil acceso en muchos casos.

Sin embargo, es precisamente desde la plena conciencia de los problemas y las limitaciones de las fuentes desde donde debemos partir para la interpretación. Y es más: creemos que esta conciencia ayuda a abrir más posibles lecturas, ya que una vez derrocado el positivismo y la ilusión de objetividad, debemos arriesgar sin 
miedo para lanzar nuevas hipótesis. Sólo así será posible no sólo escribir historia de las mujeres en el Próximo Oriente Antiguo, algo todavía absolutamente necesario, sino también escribir una historia en la que las mujeres también quepan, una historia más compleja y rica.

\section{bibliografia}

ALCOFF, Linda / POTTER, Elizabeth. Feminist Epistemologies. New York: Routledge, 1993.

ARENDT, Hannah. Entre el pasado y el futuro. Ocho ejercicios de reflexión política. Barcelona: ediciones Península, 2003.

BAHRANI, Zainab. Women of Babylon: Gender and Representation in Mesopotamia. London: Routledge, 2001.

BUDIN, Stephanie Lynn. The Myth of Sacred Prostitution in Antiquity. Cambridge / New York: Cambridge University Press, 2008.

CHAPMAN, Robert. Arqueologías de la complejidad. Barcelona: Edicions Bellaterra, 2010.

COUTO-FERREIRA, M. Érica / GARCIA-VENTURA, Agnès. Engendering Purity and Impurity in Assyriological Studies: A Historiographical Overview. Gender \& History. vol. 25, 3, pp. 513-528, 2013.

CRAWFORD, Harriet. An exploration of the world of women in third-millennium Mesopotamia. In: Women in the Ancient Near East: A Sourcebook. London / New York: Routledge, 2014, pp. 10-27.

DUNCAN, Carol. Rituales de civilización. Murcia: Nausícaä, 2007.

DURAND, Jean-Marie. L'organisation de l'espace dans le palais de Mari: le témoignage des textes. In: Le système palatial en Orient, en Grèce et à Rome, Actes du colloque de Strasbourg 19-22 juin 1985. Strasbourg: Université des sciences humaines de Strasbourg, Travaux du Centre de Recherche sur le Proche-Orient et la Grèce Antiques, 1987, pp. 39-110.

DURAND, Jean-Marie. Archives Épistolaires de Mari 1/1. Paris: Archives Royales de Mari XXVI, Éditions Recherche sur les Civilisations, 1988.

FINKELSTEIN, Jacob J. Mesopotamian Historiography. Proceedings of the American Philosophical Society. Philadelphia: American Philosophical Society, vol. 107, pp. 461-472, 1963. 
FONTANA I LÁZARO, Josep. La història després de la fi de la història. Vic: Eumo, 1992.

FONTANA I LÁZARO, Josep. La historia de los hombres: el siglo XX. Barcelona: Crítica, 2010.

FOX KELLER, Evelyn. Reflexiones sobre género y ciencia. Valencia: Alfons el Magnànim, 1991.

GARCIA-VENTURA, Agnès. Trabajar en tiempos de guerra en Mesopotamia. In: Más allá de la batalla. La violencia contra la población en el Mundo Antiguo. Zaragoza: Libros Pórtico, 2013, pp. 1-25.

GARCIA-VENTURA, Agnès. The sex-based division of work versus intersectionality: some strategies for engendering the Ur III textile work force. In: The Role of Women in Work and Society. Proceedings of the final Conference of the REFEMA project (Nanterre, 5-7 November 2014). Berlin / New York: de Gruyter, en prensa.

HARAWAY, Donna. Situated Knowledges: The Science Question in Feminism and the Privilege of Partial Perspective. Feminist Studies. vol. 14, 3, pp. 575-599, 1988.

HARAWAY, Donna. Ciencia, cyborgs y mujeres. La reinvención de la naturaleza. Madrid: Cátedra / Universitat de València, 1995.

HARDING, Sandra. Ciencia y feminismo. Madrid: ediciones Morata, 1996.

HARDING, Sandra. Feminist Standpoints. In: Handbook of Feminist Research: Theory and Praxis. Los Angeles / London: SAGE, 2012, pp. 46-64.

HARTSOCK, Nancy. The Feminist Standpoint: Developing the Ground for a Specifically Feminist Historical Materialism. In: Discovering Reality: Feminist Perspectives on Epistemology, Metaphysics, Methodology, and Philosophy of Science. Boston: Ridel, 1983, pp. 283-310.

HERNANDO, Almudena. La fantasía de la identidad. Sobre la construcción sociohistórica del sujeto moderno. Madrid: Katz Editores, 2012.

HEYD, Michael. Is there a future to the study of the Past? Culture \& History Digital Journal. vol. 1, 1, pp. 1-7, 2012.

HUIZINGA, Johan. A definition of the concept of history. In: Philosophy and History. Essays presented to Ernst Cassirer. Oxford: Clarendon Press, 1963, pp. 110 . 
JULIANO, Dolores. Las que saben. Subculturas de mujeres. Madrid: Horas y horas, 1998.

KARAHASHI, Fumi / GARCIA-VENTURA, Agnès. Overseers of Textile Workers in Presargonic Lagash, en preparación.

KNAPP, A. Bernard. Archaeology Without Gravity: Postmodernism and the Past. Journal of Archaeological Method and Theory. vol. 3, 2, pp. 127-158, 1996.

LAFONT, Bertrand. A propos de l'absence cyclique des femmes. N.A.B.U. vol. 45, pp. 24-25, 1987.

LIVERANI, Mario. Antico Oriente. Storia, società, economia. Roma: Laterza, 1988.

LIVERANI, Mario. History and Archaeology in the Ancient Near East: 150 Years of Difficult Relationship. In: Fluchtpunkt Uruk. Archälogische Einheit aus Methodischer Vielfalt. Schriften für Hans Jörg Nissen. Rahden: Verlag Marie Leidorf, 1999, pp. 1-11.

MALCOLM, X. On Afro-American History. New York: Merit Books, 1971.

MICHALOWSKI, Piotr. Sumerian. In: The Cambridge Encyclopedia of the World's Ancient Languages. Cambridge: Cambridge University Press, 2004, pp. 19-59.

MILLET-ALBÀ, Adelina. Le harem de Sîn-iqišam. In: Chagar Bazar (Syrie) III. Les trouvailles épigraphiques et sigillographiques du chantier I (2000-2002). Louvain / Paris / Dudley (MA): Peeters, 2008, pp. 239-279.

MILLETT, Kate. Sexual Politics. Urbana / Chicago: University of Illinois Press, 2000.

NELSON, Sarah Milledge. Gender in Archaeology. California: AltaMira Press, 1997.

NUÑO GÓMEZ, Laura. El mito del varón sustentador. Orígenes y consecuencias de la división sexual del trabajo. Barcelona: Icaria, 2010.

POLLOCK, Susan. Ancient Mesopotamia. The Eden that Never Was. Cambridge: Cambridge University Press, 1999.

SCOTT, Joan Wallach. Gender: A Useful Category of Historical Analysis. In: Gender and the Politics of History. New York: Columbia University Press, 1999, pp. 28-50. 
SERI, Andrea. Local Power in Old Babylonian Mesopotamia. Sheffield / Oakville: Equinox, 2012.

STOL, Marten. Women in Mesopotamia. Journal of the Economic and Social History of the Orient. vol. 38, pp. 123-144, 1995.

STOL, Marten. Vrouwen van Babylon. Prinsessen, Priesteressen, Prostituees in de bakermat van de cultuur. Utrecht: Kok, 2012.

SVÄRD, Saana. Review of "Women in the Ancient Near East", ed. Mark Chavalas, 2014. Journal of Near Eastern Studies. Chicago: University of Chicago, vol. 74, 2 , pp. 378-380, 2015.

TRINGHAM, Ruth. Casas con caras: el reto del género en los restos arquitectónicos prehistóricos. In: Arqueología y teoría feminista. Barcelona: Icaria, 1999, pp. 97-140.

VAN DE MIEROOP, Marc. Cuneiform Texts and the Writing of History. London and New York: Routledge, 1999.

WESTENHOLZ, Aage. Does Assyriology have a Future? KASKAL. Rivista di storia, ambienti e culture del Vicino Oriente Antico. Università Ca'Foscari, vol. 3, pp. 275-283, 2006.

ZIEGLER, Nele. Le harem de Zimri-Lim: La population féminine des Palais d'après les archives royales de Mari. Paris: Mémoirs de NABU, 5 / Florilegium Marianum 4, Société pour l'Étude du Proche-Orient Ancien (SEPOA), 1999. 